\title{
Empleo de arcillas uruguayas pilareadas para el blanqueo de aceite de maiz
}

\author{
Por T. Pagano, M. Sergio, A. Montenegro, W. Diano y M. A. Grompone* \\ Facultad de Química, Casilla de Correo 1157, Montevideo, Uruguay.
}

\section{RESUMEN}

Empleo de arcillas uruguayas pilareadas para el blanqueo de aceite de maiz.

En este trabajo se estudia la capacidad de adsorción de los carotenos presentes en un aceite de maíz, de una montmorillonita uruguaya modificada por diferentes procedimientos: intercambiada con iones $\mathrm{Na}^{+}$(M-Na), activada por ácido (Act), intercambiada con iones $\mathrm{Ca}^{2+}$ y pilareada con iones Keggin (Pilc-Ca), activada por ácidos y pilareada (Act Pilc-Ca) frente a dos arcillas comerciales: Tonsil Optimum de Süd-Chemie y una Montmorillonita tipo $\mathrm{K}$ 10 de Aldrich.

Los resultados indican que la muestra Act y la Pilc-Ca son tan eficientes como las comerciales pero que la capacidad de la muestra M-Na es considerablemente menor. También se efectuó ensayos con mezclas de Pilc-Ca con las arcillas comerciales, colocadas simultáneamente (en una sola etapa) o agregadas de manera sucesiva (en dos etapas). Dichos procedimientos no mejoraron la eficiencia del blanqueo.

En conclusión, la modificación Pilc-Ca permite blanquear un aceite de maíz neutralizado y desgomado a valores similares a los correspondientes a los aceites comestibles comerciales. Este efecto se mejora aún por la acción simultánea con arcillas activadas.

PALABRAS-CLAVE: Aceite de maiz - Arcillas pilareadas - Decoloración - Montmorillonita - Uruguay.

\section{SUMMARY}

Using uruguayan pillared clays for bleaching corn oil.

In the present paper adsorptive capacity to remove carotenes from a com oil by an Uruguayan montmorillonite modified by different treatments: exchange with $\mathrm{Na}^{+}$ions (M-Na), acid-activation (Act), exchange with $\mathrm{Ca}^{+2}$ and pillaring with Keggin ions (Pilc-Act), acidactivation and pillaring (Act Pil-Ca), is studied. Bleaching action of the Uruguayan modified montmorillonites is compared with two commercial clays: Tonsil Optimum (Süd-Chemie) and montmorillonite of type K 10 (Aldrich).

Our results demonstrate that Act and Pilc-Ca samples are so efficient bleaching clays as commercials but $\mathrm{M}-\mathrm{Na}$ sample is lower. Also, mixtures of Pilc-Ca with commercial clays, added together in one step or consecutively in two steps, are studied. These procedures do not improved the bleaching action.

As conclusion, the modified clay Pilc-Ca allows to bleach a neutralized and degummed corn oil, giving an end product similar to edible commercial oils. Such effect is improved with the simultaneous action with activated clays.

KEY-WORDS: Bleaching - Com oil - Montmorillonite - Pillared clays - Uruguay.

\section{INTRODUCCIÓN}

Las grasas y aceites naturales pueden contener diferentes pigmentos: clorofilas (que imparten una coloración verde), más de 70 variedades de carotenoides (que son responsables de un color amarillo/rojo), derivados de las flavinas (que contribuyen al color amariIlo), antocianidinas (cuyas sales son azules en medio básico y rojas en medio ácido aunque no se suelen encontrar en cantidades apreciables en los aceites), productos de la oxidación del gossypol (de color oscuro) y de los tocoferoles (que provocan una coloración roja oscura). El tipo y cantidad de pigmentos coloreados que posee un aceite es característico de él por lo que, en cada caso, se debe estudiar las condiciones de su blanqueo (Patterson, 1992).

Las principales materias primas utilizadas para el blanqueo de aceites son bentonitas o montmorillonitas, las que no tienen casi actividad decolorante en su estado natural. Desde el punto de vista químico, se trata de silicatos de aluminio y magnesio, y/o hierro, con un contenido variable de metales alcalino y alcalino-térreos. Por tratamiento con ácido sulfúrico o clorhídrico, se aumenta considerablemente el poder blanqueante de las arcillas, por lo que se denominan "activadas" (Kheok, 1982; Taylor, 1989; Nkpa, 1989; Habile, 1992). Algunos trabajos estudian las isotermas de adsorción tipo Langmuir o tipo Freundlich para el $\beta$-caroteno (Khoo, 1979; Morgan, 1985; Sarier, 1989; Achife, 1989; Chapman, 1992; Boki,1992; Proctor, 1996) y para la clorofila (Güler, 1992; Borghi, 1994) con arcillas activadas.

Las tierras de blanqueo actúan como donores de protones (ácidos de Brönsted) o aceptores de electrones (ácidos de Lewis). Por ejemplo, el $\beta$-caroteno se une a los sitios ácidos de la superficie de la arcilla en la forma de iones carbonio (por medio de su carbono 7) ya sea formando puentes de hidrógeno con los sitios de Brönsted o formando enlaces coordinados con sitios de Lewis (Sarier, 1989). Sin embargo, no se ha encontrado una correlación directa entre la capacidad de blanqueo y la acidez superficial. Si bien ésta contribuye a su actividad, si es muy alta puede provocar un efecto negativo por incrementar la hidrólisis del aceite y la isomerización de las cadenas alquilo de los triacilgliceroles (Morgan, 1985).

Las dimensiones aproximadas de la sección transversal de las moléculas monoméricas de caro- 
teno son $7 \times 31 \AA$ y las de las de clorofila son $12 \times 15 \AA$, lo que indica que ambas moléculas podrían éntrar en poros de radios del orden de $15 \AA$. Sin embargo, la adsorción de carotenos se obtiene usando poros de diámetros en la región de 50-100 Å mientras que para la clorofila se logra con diámetros de poros entre 50-200 $\AA$. Como éstos son considerablemente mayores que las dimensiones de las áreas transversales que corresponden a las moléculas monoméricas de ambos pigmentos, se confirma la hipótesis de Brimberg (1982) de que se encuentran como coloides dispersos en el aceite y no al estado molecular. En consecuencia, los mejores adsorbentes son aquellos que tienen, simultáneamente, adecuadas concentraciones de sitios fuertemente ácidos y alta superficie específica debida a poros cuyos diámetros se encuentran en la región de 50-200 Å (Taylor, 1989; Boki, 1992).

El pilareado también es una herramienta poderosa para variar las propiedades de las arcillas. Por medio de un intercambio iónico, se introducen cationes inorgánicos de gran tamaño, de estructura tipo

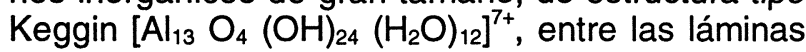
de la arcilla, lo que produce una estructura expandida. Por calcinación posterior, los cationes se convierten en óxidos, generando pilares que actúan de separadores entre dichas láminas. Además, esos pilares se unen covalentemente con la arcilla y el espacio interlamelar queda constante, perdiendo así la montmorillonita la propiedad de expandirse frente al agua y los glicoles. Muy pocos son los trabajos publicados sobre el uso de arcillas pilareadas en el blanqueo de aceites (Taylor, 1989; Mokaya, 1993).

En este trabajo se estudia la capacidad de adsorción de carotenos de una montmorillonita uruguaya modificada por diferentes procedimientos (intercambio con iones $\mathrm{Na}$, activación por ácido y/o pilareado) frente a otras arcillas blanqueadoras comerciales. La montmorillonita uruguaya utilizada como materia prima fue caracterizada por Ford (1989). Se trata de un mineral poco compactado con $80-90 \%$ de montmoriIlonita cálcica (con algo de $\mathrm{Na}, \mathrm{K}, \mathrm{Fe}$ y $\mathrm{Mn}, 5-15 \%$ de arena y $5 \%$ de carbonato de calcio).

\section{PARTE EXPERIMENTAL}

Como materia prima se utilizó un aceite de maíz uruguayo extraído, neutralizado y desgomado a nivel industrial. Se eligió un aceite de maíz ya que se comercializa corrientemente en el Uruguay y presenta una fuerte coloración amarillo-rojiza. Sus principales pigmentos son xantofilas y carotenos, con un contenido casi nulo de clorofila (Patterson, 1992).

Las condiciones de blanqueo en el laboratorio se eligieron de acuerdo con la bibliografía (Patterson, 1992; Hodgson, 1996): temperatura de $100^{\circ} \mathrm{C}$, vacío de $480 \mathrm{~mm}$ de $\mathrm{Hg}$ y un tiempo de 15 minutos. Luego de enfriar la mezcla de aceite y arcilla a temperatura ambiente bajo vacío, se agregó un ayudante de filtración (Celite 521) y se filtró a vacío. Todos los ensayos se realizaron, como mínimo, por duplicado.

Los adsorbentes ensayados fueron modificaciones de la Montmorillonita uruguaya (muestra $\mathrm{M}$ ):

a) la arcilla natural $\mathrm{M}$ activó a reflujo durante 2 horas con $\mathrm{H}_{2} \mathrm{SO}_{4} 3 \mathrm{M}$ (denominada Act)

b) la muestra $\mathrm{M}$ se intercambió con $\mathrm{NaCl}$, con lo que se separó la fracción de arcilla que está parcialmente cementada. Se secó a estufa a $100^{\circ} \mathrm{C}$ (denominada $\mathrm{M}-\mathrm{Na}$ ).

c) la muestra activada según a) se pilareó con polioxocationes de $\mathrm{Al}_{13}$ y se calcinó durante 2 horas a $550^{\circ} \mathrm{C}$, dando un sólido denominado Act Pilc-Ca (PILC = pillared interlayered clays)

d) la muestra natural $\mathrm{M}$ se intercambió con $\mathrm{CaCl}_{2}$, se pilareó según c) y se calcinó durante 2 horas a $550^{\circ} \mathrm{C}$, dando un sólido poroso denominado Pilc-Ca.

La técnica empleada para pilarear se encuentra en la literatura (Diano, 1994). Se preparó una solución pilareante dejando gotear lentamente una solución $0.2 \mathrm{M}$ de $\mathrm{NaOH}$ sobre una solución $0.2 \mathrm{M}$ de $\mathrm{AlCl}_{3}$ fuertemente agitada, de tal modo que la relación molar final OH/Al fue 2.0 y el pH de 3.95. La mezcla resultante se envejeció una hora a $50^{\circ} \mathrm{C}$ y sobre ella se agregó lentamente una suspensión del $14 \%$ de la montmorillonita cálcica con fuerte agitación y en cantidad tal que se obtuvo una relación final Al/arcilla de $5 \mathrm{mmoles} / \mathrm{gramo}$. Al final del agregado de la suspensión de arcilla se ajustó el pH con una solución de $\mathrm{NH}_{4} \mathrm{Cl}$ hasta 5.5 y luego se envejeció tres horas a $80^{\circ} \mathrm{C}$. El sólido obtenido se filtró a vacío, lavándolo con agua destilada caliente hasta que la conductividad fue de 20 microSiemens/cm. La PILC resultante fue secada en estufa a $100^{\circ} \mathrm{C}$ y luego calentada a una velocidad de $1^{\circ} \mathrm{C}$ por minuto hasta $550^{\circ} \mathrm{C}$, manteniendo luego esa temperatura durante dos horas. El área BET del sólido Pilc-Ca fue de $360 \mathrm{~m}^{2} / \mathrm{g}$ y la altura de los poros, determinada por Rayos X, de $0.75 \mathrm{~nm}$.

A los efectos comparativos, también se utilizó dos tierras de blanqueo comerciales:

1) Tonsil Optimum (Süd-Chemie AG), denominada $T$. Se trata de una arcilla altamente activada y particularmente recomendada para aceites de blanqueo difícil (Patterson, 1992) que, de acuerdo con el boletín técnico que suministra el fabricante, presenta un área superficial específica de $190 \mathrm{~m}^{2} / \mathrm{g}$, una acidez residual ( $\mathrm{NaOH} \%$ en peso) de 0.25 y un pH (de la suspensión al 10\%) de 3.5.

2) Montmorillonita tipo $\mathrm{K} 10$ (Aldrich Chemical Co.), denominada $A$. De acuerdo con el boletín técnico de la empresa Aldrich, su área superficial específica es de $20-40 \mathrm{~m}^{2} / \mathrm{g}$.

A los efectos comparativos también la tierra de blanqueo de Aldrich (A) se pilareó usando la técnica descrita, denominándola A-Pilc.

La eficiencia del blanqueo se determinó por medida del color residual del aceite (según técnica AOCS Cc 
13b-50) en un tintómetro manual Lovibond modelo $E$ (The Tintometer $L$ td.), en celdas de vidrio de 5,25 pulgadas de espesor. (En este trabajo se informan los valores promedios obtenidos en base a las repeticiones realizadas de cada ensayo). Como referencia de color se tomó la del aceite de maíz refinado comestible (o sea, neutralizado, blanqueado y desodorizado) que la misma empresa industrial que suministró el aceite para los ensayos de blanqueo, comercializa en el Uruguay.

\section{RESULTADOS Y DISCUSIÓN}

En la tabla I se resumen los resultados obtenidos con la montmorillonita activada (Act) en diferentes concentraciones en comparación con los obtenidos con A y con $\mathrm{T}$ al $2 \%$. En esta tabla también se indica el color inicial del aceite neutralizado usado en dichos ensayos y el color del aceite de maíz comestible tomado como referencia. A iguales concentraciones, la arcilla uruguaya activada es tan eficiente como las comerciales.

Tabla I

Blanqueos con diferentes concentraciones de la montmorillonita uruguaya activada por ácido (Act)

\begin{tabular}{lcc}
\hline \multicolumn{1}{c}{ Ensayos } & Amarillo & Rojo \\
\hline Aceite comestible comercial & 26 & 3.7 \\
Aceite neutralizado a blanquear & 30 & 5.6 \\
Aceite blanqueado con: & & \\
T2\% & 20 & 2.1 \\
A2\% & 20 & 2.0 \\
Act $0.5 \%$ & 30 & 5.0 \\
Act $1.0 \%$ & 24 & 4.0 \\
Act $2.0 \%$ & 20 & 2.0 \\
\hline
\end{tabular}

La activación ácida es un procedimiento corriente utilizado para mejorar las propiedades adsorbentes de las arcillas. En consecuencia, es de interés estudiar también el efecto de otras modificaciones sobre esa misma arcilla. En la Tabla II se indican los resultados de los ensayos de blanqueo realizados con la montmorillonita uruguaya intercambiada con iones $\mathrm{Na}^{+}(\mathrm{M}-\mathrm{Na})$, comparados con los realizados con las arcillas de referencia $A$ y $T$. En dicha tabla también se indica el color inicial del aceite neutralizado usado en dichos ensayos y el color del aceite de maíz comestible tomado como referencia. Se ve que los colores del aceite blanqueado con Tonsil (T) al $2 \%$ o con la arcilla de Aldrich (A) al $2 \%$ son ligeramente mejores que los del aceite de maíz comestible que se comercializa en el Uruguay. Sin embargo, todos los blanqueos realizados con las modificaciones de la montmorillonita uruguaya (M-Na) son considerablemente menos eficientes, por lo que se descarta el empleo de este tipo de modificación.
Tabla II

Blanqueos con la montmorillonita uruguaya intercambiada con iones $\mathrm{Na}$ (M-Na)

\begin{tabular}{lcr}
\hline \multicolumn{1}{c}{ Ensayos } & Amarillo & Rojo \\
\hline Aceite comestible comercial & 26 & 3.7 \\
Aceite neutralizado a blanquear & 26 & 10.3 \\
Aceite blanqueado con: & & \\
T2\% & 20 & 3.5 \\
A2\% & 19 & 3.1 \\
M-Na 2\% & 26 & 11.3 \\
M-Na 3\% & 24 & 8.5 \\
\hline
\end{tabular}

A los efectos de establecer si el pilareado puede ser un procedimiento que mejore la capacidad de blanqueo, se aplicó tanto sobre la arcilla uruguaya como sobre la muestra comercial A. Los resultados se muestran en la tabla III. Se observa que el blanqueo realizado con Pilc$\mathrm{Ca}$ al $2 \%$ es casi tan eficiente como el realizado con las muestras comerciales $\mathrm{A}$ o $\mathrm{T}$ en iguales concentraciones. El blanqueo realizado con A-Pilc al $2 \%$ es aún mejor. De ello se deduce que el pilareado puede ser un método adecuado para mejorar la capacidad de blanqueo de la montmorillonita uruguaya. Estos resultados son diferentes de los obtenidos por Taylor (1989) ya que en dicho trabajo concluye que las montmorillonitas cálcicas activadas por ácido son muy superiores a las pilareadas y activadas y algo mejores que las sódicas activadas, a los efectos de adsorber carotenos.

Tabla III

Blanqueos con las arcillas pilareadas Pilc-Ca y A-Pilc

\begin{tabular}{lcc}
\hline \multicolumn{1}{c}{ Ensayos } & Amarillo & Rojo \\
\hline Aceite comestible comercial & 26 & 3.7 \\
Aceite neutralizado a blanquear & 29 & 6.4 \\
Aceite blanqueado con: & & \\
T2\% & 25 & 3.6 \\
A2\% & 25 & 3.4 \\
A-Pilc 2\% & 25 & 2.6 \\
Pilc-Ca 2\% & 25 & 3.8 \\
\hline
\end{tabular}

A los efectos de mejorar más la eficiencia del blanqueo realizado con la arcilla Pilc-Ca, se efectuó ensayos con mezclas de ella con las arcillas comerciales, colocadas simultáneamente (en una sola etapa). Los resultados obtenidos se informan en la tabla IV. El blanqueo realizado con un agregado simultáneo de $\mathrm{A} 1 \%$ y Pilc-Ca $1 \%$ es más eficiente que el realizado tanto con $\mathrm{A}$ al $2 \%$ como con Pilc-Ca al $2 \%$. Esto puede indicar que son diferentes las especies que 
se adsorben en cada tipo de blanqueador. Esto no es de extrañar pues, de acuerdo con Taylor (1989), los carotenos actúan como si estuvieran bajo forma coloidal dispersos en el aceite y no como moléculas monoméricas, por lo que pueden presentar diferencias de comportamiento.

Tabla IV

Blanqueos con mezclas de la montmorillonita uruguaya pilareada (Pilc-Ca) y de las arcillas comerciales A O T

\begin{tabular}{lcc}
\hline \multicolumn{1}{c}{ Ensayos } & Amarillo & Rojo \\
\hline Aceite comestible comercial & 26 & 3.7 \\
Aceite neutralizado a blanquear & 29 & 6.4 \\
Aceite blanqueado con: & & \\
T2\% & 25 & 3.6 \\
A2\% & 25 & 3.4 \\
Pilc-Ca 2\% & 25 & 3.8 \\
A 1\% + Pilc-Ca 1\% & 23 & 2.0 \\
A 2\% + Pilc-Ca 2\% & 20 & 1.6 \\
T 2\% + Pilc-Ca 2\% & 21 & 1.7 \\
\hline
\end{tabular}

También se realizó un blanqueo con las mismas cantidades de arcillas referidas anteriormente, pero agregadas de manera sucesiva (en dos etapas). Esta operación consistió en blanquear dos veces la muestra, como si cada etapa fuera independiente (es decir, blanquear una primera vez con $A$ al $1 \%$, enfriar a vacío, filtrar y volver a blanquear pero con Pilc-Ca al 1\%). En la tabla V se indican los resultados obtenidos. Se concluye que este procedimiento no mejoró la eficiencia del blanqueo respecto al realizado en iguales proporciones de arcillas, pero en una sola etapa.

Tabla V

Blanqueos en dos etapas con la montmorillonita uruguaya pilareada (Pilc-Ca) y con la arcilla comercial A

\begin{tabular}{|c|c|c|}
\hline Ensayos & Amarillo & Rojo \\
\hline Aceite comestible comercial & 26 & 3.7 \\
\hline Aceite neutralizado a blanquear & 29 & 6.4 \\
\hline \multicolumn{3}{|l|}{ Aceite blanqueado } \\
\hline \multicolumn{3}{|l|}{ en una etapa: } \\
\hline T 2\% & 25 & 3.6 \\
\hline A $2 \%$ & 25 & 3.4 \\
\hline A $1 \%+$ Pilc-Ca $1 \%$ & 23 & 2.0 \\
\hline \multicolumn{3}{|l|}{ en dos etapas. } \\
\hline 1. ${ }^{\text {etapa }}$ & & \\
\hline Pilc-Ca 1\% & 21 & 2.7 \\
\hline
\end{tabular}

Como último procedimiento, se blanqueó con la montmorillonita uruguaya activada y pilareada (Act Pilc-Ca) al $2 \%$ y se comparó con el blanqueo realizado en una etapa con una mezcla de la Act al $1 \%$ y la Act Pilc-Ca al 1\%. Los resultados se informan en la tabla VI. Dichos procedimientos no mejoran la eficiencia del blanqueo.

Tabla VI

Blanqueos con mezcla de la montmorillonita uruguaya activada (Act) y de la montmorillonita uruguaya activada y pilareada (Act Pilc-Ca), en una etapa

\begin{tabular}{lcc}
\hline \multicolumn{1}{c}{ Ensayos } & Amarillo & Rojo \\
\hline Aceite comestible comercial & 26 & 3.7 \\
Aceite neutralizado a blanquear & 30 & 5.6 \\
Aceite blanqueado con: & & \\
T 2\% & 20 & 2.1 \\
A 2\% & 20 & 2.0 \\
Act 2\% & 20 & 2.0 \\
Act Pilc-Ca 2\% & 25 & 2.0 \\
Act 1\%+ Act Pilc-Ca 1\% & 21 & 2.0 \\
\hline
\end{tabular}

De todos los resultados obtenidos se concluye que la modificación cálcica pilareada obtenida a partir de una montmorillonita uruguaya permite blanquear un aceite de maíz neutralizado y desgomado a valores de color rojo Lovibond similares a los correspondientes a los aceites comestibles comerciales. Este efecto se mejora aún por la acción simultánea con arcillas activadas del tipo $\mathrm{K} 10$ de Aldrich, Tonsil Optimum o la uruguaya Act.

\section{AGRADECIMIENTOS}

A la QF Selva Pombo de la Empresa COUSA, por el suministro de las muestras de aceite de maíz; a las Ings. Químs. Mayra Valle y Mariella Chiappe por las determinaciones de color.

\section{BIBLIOGRAFÍA}

Achife, E. C. e Ibemesi, J. A. (1989). - «Applicability of the Freundlich and Langmuir adsorption isotherms in the bleaching of rubber and melon seed oils».- J. Am. Oil Chem. Soc. 66 (2), 247-252

Aldrich Chemical Co. - «Mineral adsorbents, filter agents and drying agents".- Technical Information Bulletin AL-143.

American Oil Chemists' Society (1989). - «Official Methods and Recommended Practices".- 4. a Edición, R. E. Walker (Ed.).- American Oil Chemists' Society, Champaign (IL). 
Boki, K., Kubo, M., Kawasaki, N. y Mori, H. (1992). «Adsorption isotherms of pigments from alkali-refined vegetable oils with clay minerals".- J. Am. Oil Chem. Soc. 69 (4), 372-378.

Borghi, A. B. M. P. y Barrera-Arellano, D. (1994). - «Remoção de clorofila por argilas ativadas".- Espuma, 10 (25), 49-51.

Brimberg, U. I. (1982). - «Kinetics of bleaching of vegetable oils".- J. Am. Oil Chem. Soc. 59 (2), 74-78.

Chapman, D. M. y Pfannkoch, E. A. (1992). - «Thermodynamic limitations for pigment adsorption during the bleaching of triglyceride oils".- J. Am. Oil Chem. Soc. 69 (10), 1009-1014.

Diano, W., Rubino, R. y Sergio, M. (1994). -«Al-pillared montmorillonite: preparation from concentrated slurries of homoionic $\mathrm{Ca}$ clay, characterization and thermal stability".- Microporous Materials 2, 179-184.

Ford, I. y Sergio, M. (1989). - «Caracterización mineralógica y origen probable de la bentonita de Bañado de Medina Fm formación Yaguarí Ps".- Boletín de Investigación N. ${ }^{\circ} 23$, Facultad de Agronomía, Montevideo.

Güler, Ç. y Tunç, F. (1992). - "Chlorophyll adsorption on acid-activated clay».- J. Am. Oil Chem. Soc. 69 (9), 948-950.

Habile, M., Barlow, P. J. y Hole, M. (1992). -«Adsorptive bleaching of soybean oil with non-montmorillonite Zambian clays".- J. Am. Oil Chem. Soc. 69 (4), 379-383.

Hodgson, A. S. (1996). - «Refining and Bleaching» en «Bailey's Industrial Oil \& Fat Products».- . $^{\text {a }}$ edición, Vol. 4, Cap. 3. Y. H. Hui, (Ed.).- John Wiley \& Sons, Inc., New York.

Kheok, S. C. y Lim, E. E. (1982). - «Mechanism of palm oil bleaching by montmorillonite clay activated at various acid concentrations".- J. Am. Oil Chem. Soc. 59 (3), 129-131.
Khoo, L. E., Morsingh, F. y Liew, K. Y. (1979). -«The adsorption of $\beta$-carotene I. By bleaching earths".- J. Am. Oil Chem. Soc. 56 (7), 672-675.

Mokaya, R., Jones, W., Davies, M. E. y Whittle, M. E. (1993). - «Chlorophyll adsorption by alumina-pillared acid-activated clays".- J. Am. Oil Chem. Soc. 70 (3), 241-244.

Morgan, D. A., Shaw, D.B., Sidebottom, M. J., Soon, T. C. y Taylor, R. S. (1985). - "The function of bleaching earths in the processing of palm, palm kernel and coconut oils".- J. Am. Oil Chem. Soc. 62 (2), 292-299.

Nkpa, N. N., Arowolo, T. A. y Akpan, H. J. (1989). - «Quality of Nigerian palm oil after bleaching with local treated clays". - J. Am. Oil Chem. Soc. 66 (2), 218-222.

Patterson, H. B. W. (1992). - -Bleaching and purifying fats and oils:Theory and Practice».- AOCS Press, Champaign (IL).

Proctor, A. y Toro-Vázquez, J. F. (1996). - «The Freundlich isotherm in studying adsorption in oil processing". $-\mathrm{J}$. Am. Oil Chem. Soc. 73 (12), 1627-1632.

Sarier, N. y Güler, Ç. (1989). - «The mechanism of $\beta$-carotene adsorption on activated montmorillonite".- J. Am. Oil Chem. Soc. 66 (7), 917-923.

Süd-Chemie A. G. «Highly active bleaching earths: TONSIL».- Boletín Técnico, München.

Taylor, D. R., Jenkins, D. B. y Ungermann, C. B. (1989). «Bleaching with altemative layered minerals: a comparison with acid-activated montmorillonite for bleaching soybean oil».-J. Am. Oil Chem. Soc. 66 (3), 334-341.

Recibido: Septiembre 1997 Aceptado: Noviembre 1997 